\title{
Fli1 deficiency promotes CXCL13 expression from macrophages, contributing to the development of systemic sclerosis
}

Takashi Taniguchi, Yoshihide Asano, Takashi Yamashita, Kouki Nakamura, Ryosuke Saigusa, Yohei Ichimura, Takehiro Takahashi, Tetsuo Toyama, Ayumi Yoshizaki, Shinichi Sato.

Dermatology, University of Tokyo Graduate School of Medicine, Tokyo, Japan.

\section{INTRODUCTION}

Systemic sclerosis (SSc) is a multisystem autoimmune and vascular disease resulting in extensive organ fibrosis. CXCL13 induces chemotaxis of mature B cells, follicular helper T cells, T helper (Th) 17 cells, and regulatory T cells through its receptor CXCR5, while inhibiting angiogenesis by interfering with basic fibroblast growth factor. To investigate the role of CXCL13 in SSc, we examined the influence of Fli1 deficiency, a potential predisposing factor of this disease, on CXCL13 expression and assessed clinical correlation of serum CXCL13 levels by multivariate regression analysis.

\section{METHODS}

\section{Gene silencing of $F L / 1$ and $\mathrm{qRT}-\mathrm{PCR}$}

Cells were transfected with $10 \mathrm{nM}$ of FL/1 siRNA or scrambled nonsilencing RNA using HiPerfect transfection reagent for 72 hours. mRNA levels of the FLI1 and CXCL13 genes in human cells and those of the Fli1 and $C x c / 13$ genes in murine macrophages were examined by qRTPCR and normalized to mRNA levels of the GAPDH or Gapdh genes. The measurement of serum CXCL13 levels

Specific ELISA kits were used to measure serum CXCL13 levels in 56 SSc patients ( 52 women, 4 men; age, median [ 25 - 75 percentile], 59 years [51.5 - 69]; disease duration, 5 years [2 - 13]) after getting informed consent.

\section{RESULTS}
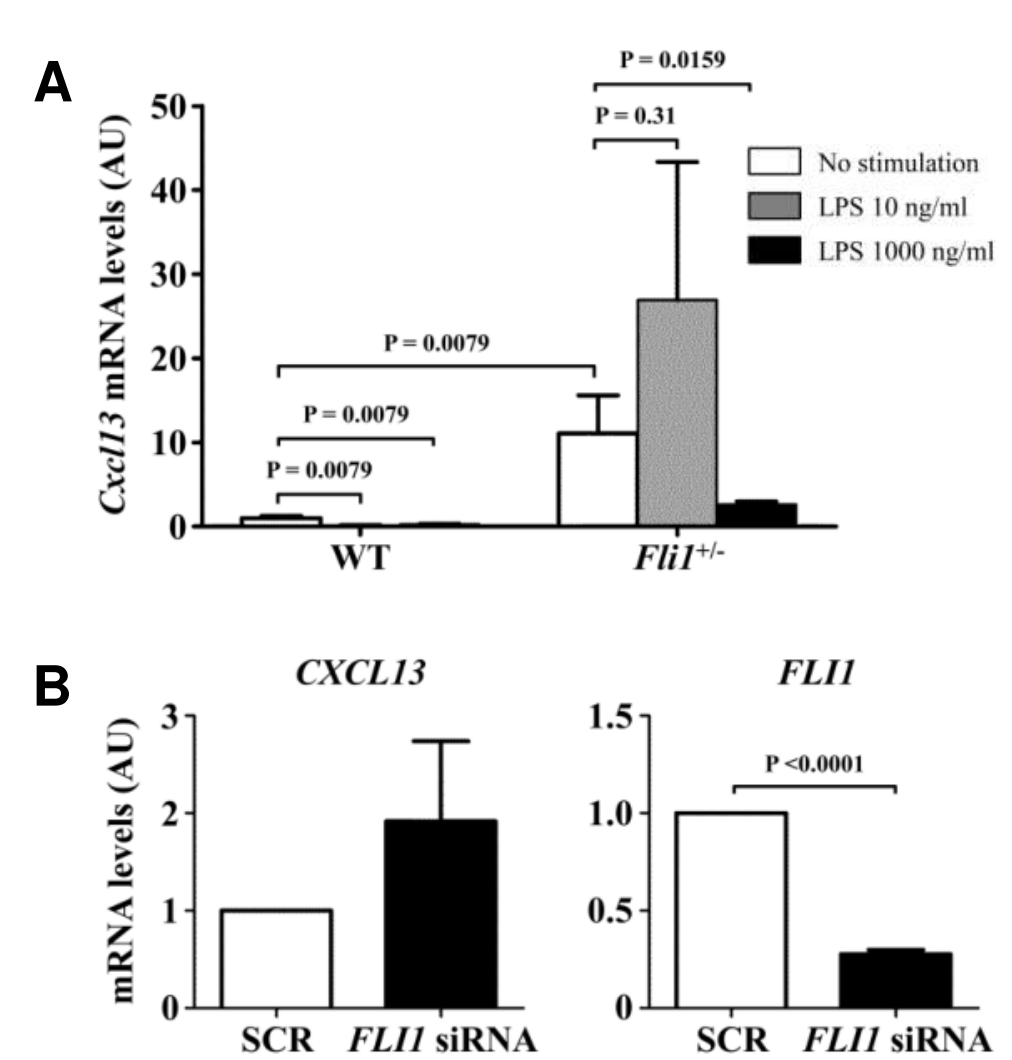

C
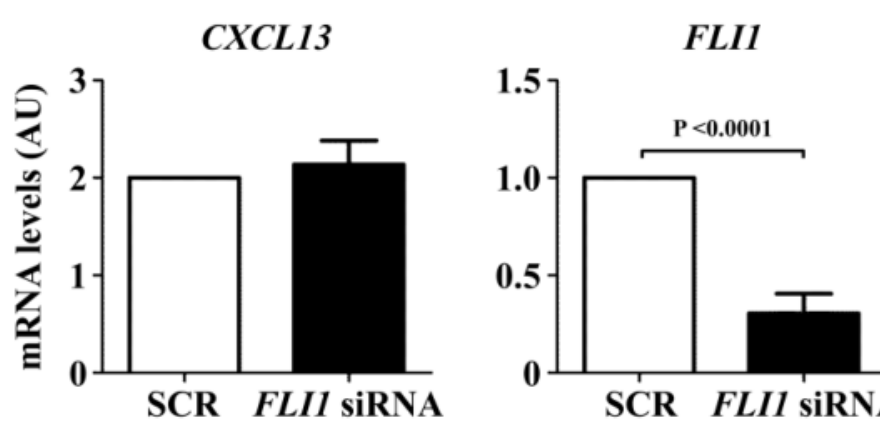

Figure 2. Serum CXCL13 levels are elevated in SSc patients, especially in those with diffuse cutaneous involvement and interstitial lung disease.

(A) Serum CXCL13 levels were measured in diffuse cutaneous SSc (dcSSc) patients, limited cutaneous SSc (IcSSc) patients, and healthy controls. Statistical analysis was carried out with one-way ANOVA followed by Turkey post hoc test $\left({ }^{*} P<0.05\right)$. (B) Serum CXCL13 levels were compared between SSc patients with interstitial lung disease (ILD) and those without. Statistical analysis was carried out with Mann-Whitney U-test.

Figure 1. Fli1 deficiency regulates CXCL13 expression in macrophages.

(A) Cxc/13 mRNA levels were determined by qRT-PCR in murine Fli ${ }^{+-}$and wild type (WT) peritoneal macrophages treated or untreated with lipopolysaccharide $(n=5)$. (B, C) CXCL13 mRNA expression was evaluated by $q R T-P C R$ in human dermal fibroblasts $(n=8, B)$ and human dermal microvascular endothelial cells (HDMECs; $n=$ $4, C)$ treated with FLI1 siRNA or (SCR). Results of controls or relative value compared with the controls are expressed as means \pm SEM. Statistical analysis was carried out with Mann-Whitney U-test. Statistical significance was determined after Bonferroni correction in experiments with macrophages. scrambled non-silencing RNA
A

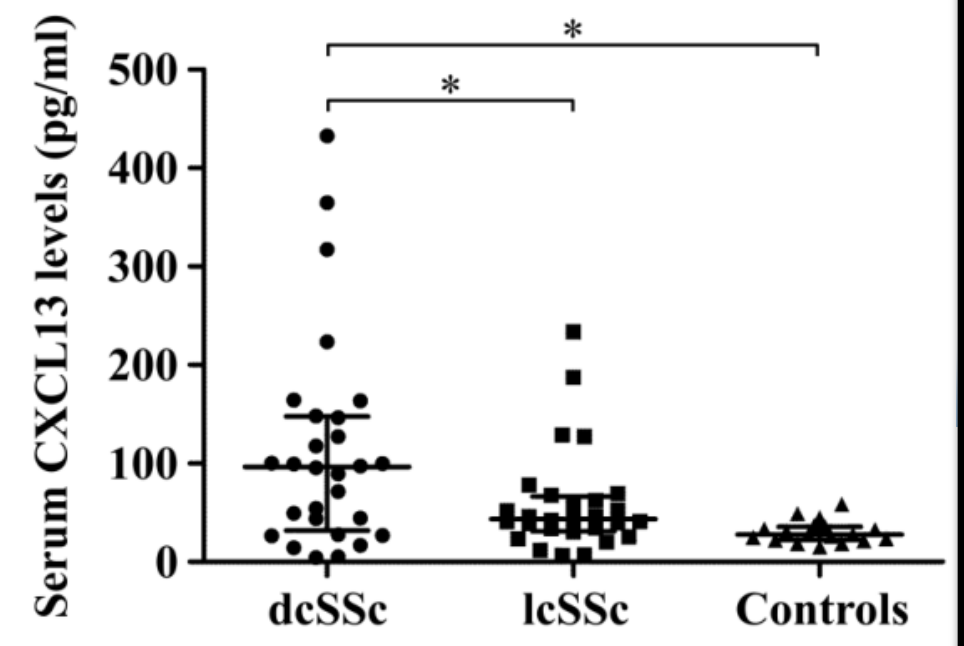

Figure 3. Serum CXCL13 levels correlate with mRSS and pulmonary function test results (\%VC and \%DLco).

(A-C) Serum CXCL13 levels correlated positively with modified Rodnan total skin thickness score (mRSS, A) and negatively with the percentage of predicted vital capacity $(\% \vee C, B)$ and the percentage of predicted diffusion lung capacity for carbon monoxide (\%DLco, C). Statistical analysis was carried out with Spearman's rank correlation test.

A

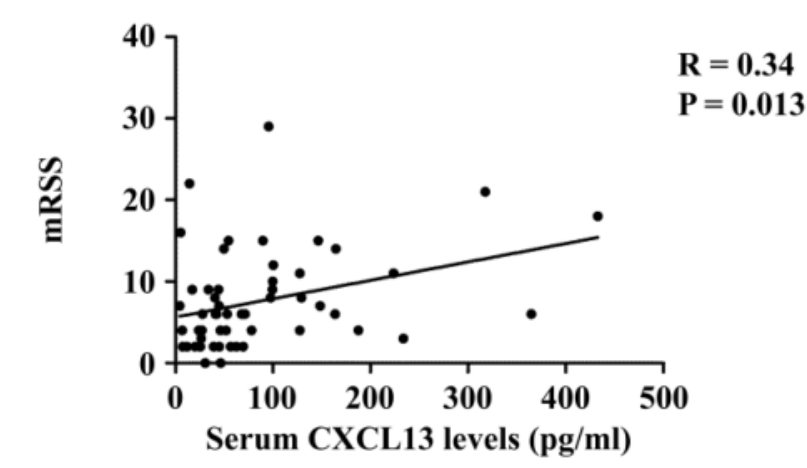

B

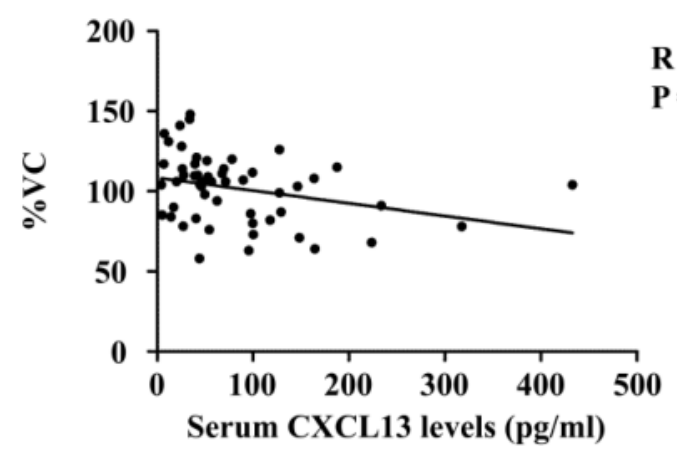

C

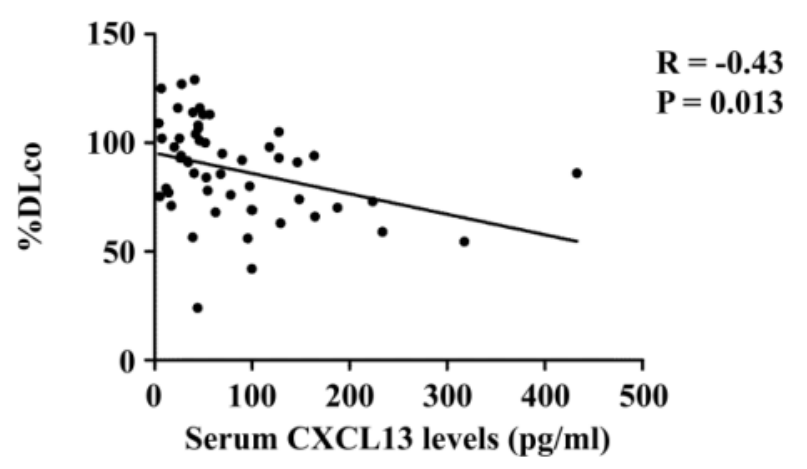

Table 1. Clinical correlation of serum CXCL13 levels in SSc

\begin{tabular}{|l|c|c|l|}
\hline \multirow{2}{*}{} & \multicolumn{2}{|c|}{ Serum CXCL13 levels } & \\
\cline { 2 - 4 } & $\begin{array}{c}\text { Elevated } \\
(\mathrm{n}=27)\end{array}$ & $\begin{array}{c}\text { Not elevated } \\
(\mathrm{n}=29)\end{array}$ & P values \\
\hline Sex, no. male : no. female & $2: 25$ & $2: 27$ & 1.0 \\
\hline Age, mean years & $59(54-70)$ & $58(49.5-69)$ & 0.62 \\
\hline Disease duration, mean years & $7.5(3-20)$ & $3(1.5-10)$ & 0.034 \\
\hline Skin sclerosis (dcSSc: IcSSc) & $18: 9$ & $10: 19$ & 0.032 \\
\hline Clinical features, \% & & & \\
\hline Pitting scars & $48(13 / 27)$ & $7(2 / 29)$ & 0.0007 \\
\hline Digital ulcers & $63(17 / 27)$ & $7(2 / 29)$ & $<0.01$ \\
\hline Raynaud's phenomenon & $100(27 / 27)$ & $72(21 / 29)$ & 0.0046 \\
\hline Telangiectasia & $67(18 / 27)$ & $33(9 / 27)$ & 0.029 \\
\hline Nailfold bleeding & $63(17 / 27)$ & $69(20 / 29)$ & 0.78 \\
\hline Organ involvements, \% & & & \\
\hline Esophageal dysfunction & $81(22 / 27)$ & $66(19 / 29)$ & 0.23 \\
\hline Interstitial lung disease & $78(21 / 27)$ & $41(12 / 29)$ & 0.0073 \\
\hline Decreased \%DLco & $36(9 / 25)$ & $7(2 / 28)$ & 0.016 \\
\hline Decreased \%VC & $27(7 / 26)$ & $7(2 / 28)$ & 0.072 \\
\hline Elevated RVSP & $26(7 / 27)$ & $7(2 / 29)$ & 0.073 \\
\hline Heart involvement & $37(10 / 27)$ & $0(0 / 29)$ & 0.0002 \\
\hline Scleroderma renal crisis & $11(3 / 27)$ & $0(0 / 29)$ & 0.11 \\
\hline Laboratory findings, \% & & & \\
\hline Elevated serum IgG & $30(8 / 27)$ & $4(1 / 27)$ & 0.024 \\
\hline Elevated ESR & $60(15 / 25)$ & $35(9 / 26)$ & 0.095 \\
\hline Increased CRP & $26(7 / 27)$ & $24(7 / 29)$ & 1.0 \\
\hline
\end{tabular}

Table 2. Factors predicting serum CXCL13 levels determined by multivariate regression analysis.

\begin{tabular}{|l|c|c|c|}
\hline & Estimate & Standard error & $\mathrm{P}$ value \\
\hline Intercept & 28.4 & 18.2 & $\mathrm{P}=0.12$ \\
\hline Digital ulcers & 53.4 & 22.3 & $\mathrm{P}=0.020$ \\
\hline Interstitial lung disease & 67.2 & 21.5 & $\mathrm{P}=0.0029$ \\
\hline
\end{tabular}

\section{CONCLUSION}

CXCL13 expression is up-regulated by Fli1 deficiency in macrophages, potentially contributing to the development of tissue fibrosis,

vasculopathy, and immune activation in SSc, especially ILD and digital ulcers.

This pathological process may be mediated by a multifaceted role of CXCL13, such as the regulation of macrophage/B cell interaction, Th17 cell infiltration, and angiogenesis.

The association of Fli1 deficiency with CXCL13 up-regulation in macrophages further supports the notion that Fli1 deficiency is a critical predisposing factor of SSc.

\section{Conflict of interest}

The authors declare no conflict of interest related to this study. 